\title{
Research and Design of UUV Navigation and Control Integrative Simulation System Based on Component ${ }^{*}$
}

\author{
Shengjie Wang, Fengju Kang \\ Underwater Information and Control State Key Lab, Marine College of Northwestern Polytechnical University, Xi'an, China \\ Email: xxm1960@126.com
}

Received April 21, 2012; revised May 21, 2012; accepted June 5, 2012

\begin{abstract}
This paper uses the component-based technology and the object oriented simulation technology to analyze the UUV navigation and control integration simulation system. We divide the system into components based on its structure, and describe every component using active diagram. By using the component-based technology, the system described here is easier to extended and be reused. At last, it realizes the whole UUV integrated navigation simulation course using the system to validate the availability.
\end{abstract}

Keywords: UUV; Integrative Simulation; Component

\section{Introduction}

In order to increase the efficiency of the simulation software development observably, using object oriented technology only during the developing process is not enough; the existent simulation component should be used in simulation program construction. The idea of component based technology is to divide the application of the complex system into some small and simplex components which can be used in different develop environment. By using the component technology, the system development can be translated into the development and integration of the components, and along with the accumulate of the component, the proportion of the development is decreasing while the proportion of integration is increasing in the develop process of software, therefore, the efficiency of the software development is increased greatly but the cost is decreased.

The component based simulation is a very effective form of the simulation software reuse, and lots of researchers are worked at the modeling methods of the universal component in different area, for example, Paredis [1] proposed a combined simulation method based on component. Changjiang Wan [2] put a modeling method of component prototype based on semantics forward to predigest the unit simulation environment modeling process. Peng Wang [3] advanced the system construction and the realize method of the CGF according to simulation component, which can create kinds of force model flexibly and suit different operation platform.

${ }^{*}$ National key laboratory of underwater information process and control Foundation (9140C2305041001).
UUV navigation and control system is composed by inertial navigation part, Doppler speed meter, GPS receiver, integrated navigation system and control system etc. For the purpose of reuse the simulation model conveniently, the model is encapsulated into component according to its function and demand, then, the UUV navigation and control system is constructed to realize the integrative function and provide a digital simulation supporting environment for the research of UUV.

\section{System Framework}

The main function of the navigation and control integrative simulation system is to simulate the process that UUV under the function of SINS and take DVL as assistant navigation at the settled depth, then after stated time of voyage, climbing to certain depth to modify the data by receiving GPS signal. Figure 1 shows the framework of the system, which include five parts: UUV dynamics and kinematics calculation, multi navigation sensor data simulation, integrated navigation data fusion, control system simulation, data-base management.

The UUV dynamics and kinematics module receives rudder information to accomplish UUV movement calculation and produce the trail data; the function of the multi navigation sensor data simulation module is to produce each navigation sensor's data and its navigation calculation; the integrated navigation data fusion module achieves the pretreatment of the navigation data, the estimate and measurement of the public state and the federal filter of the state information; the control system simulation includes the transform of navigation parameter, the control 


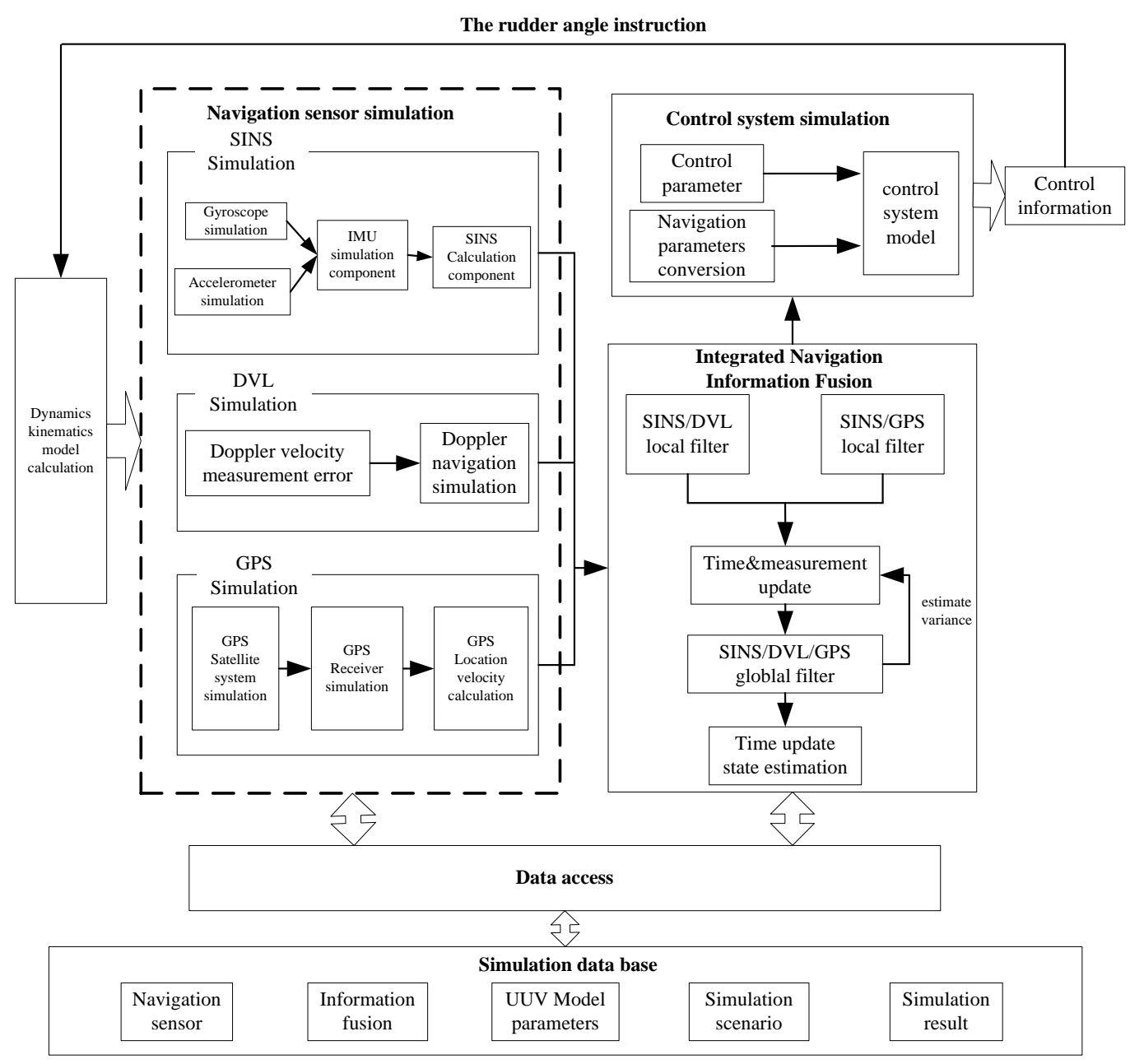

Figure 1. Integrative simulation system framework.

system setting and the control model calculation; the simulation data-base model composes the data of navigation sensor parameter, information fusion parameter, UUV model parameter, simulation scenario information and the simulation result.

\section{The Design of Navigation and Control Integrative Simulation System Based on Component}

According to its function and construction, the UUV navigation and control integrative simulation system can be divided into components showed in Figure 2.

IMU Simulator is the simulation component of the inertial measurement unit; DVL Simulator is the simulation component of Doppler speed meter; GPS Simulator is the simulation component of the GPS. they encapsulated relevant navigation system model. SINS Navigation is the calculation simulation component of the strap-down inertial navigation; it can calculate UUV's navigation information such as position, velocity, attitude, based on the angle velocity and acceleration information supplied by
IMU. Combo Navigation is the integrated navigation simulation component which can realize the information fusion of all the navigation systems and get the integrated navigation information. Control System is the component of the UUV's control system that in charge of UUV movement control based on the integrated navigation information provided by integrated navigation component. Kinetic Model is the kinetics and kinematics simulation component of UUV, which is used to calculate its movement. The detailed description of the structure and work flow of the IMU simulation component, SINS calculation simulation component, DVL simulation component, GPS simulation component and the integrated navigation simulation component is as follows, along with the component active diagram.

\subsection{IMU Simulation Component}

IMU simulation component can simulate the main error of inertial navigation system component with diversified precision, and create the measure data of the inertial device according to the given UUV trail and the error char- 
acter of the inertial device. IMU simulation component is structured by four levels: first, component interface level, including trail information input interface (ITrail), event information interactive interface (IEvent), gyroscope information output interface (IGyroscope), accelerator information output interface (IAccelerator), initialization information interface (IInitialization); second, component setting level, including the setting of accelerator error parameter (ParaAcc), gyroscope error parameter (ParaGyro), device scale parameter (ParaScale) and the time parameter (ParaTime); the third one is model calculation level that makes up of math function class (MathFunction), noise produce class (NoiseClass), frame transform class (FrameClass), IMU sensor error simulation class (IMUSensorErrClass), IMU sensor data create class (IMUSensor-
Class); and the last level is run management level that is composed by thread function (ThreadFunc), timer function (TimerFunc)and simulation operation class (SimRunClass).

The IMU simulation component active diagram is showed in Figure 3.

After the start of the simulation component, the first thing is to finish the IMU error model parameter setting, then enter the simulation circle and request time promoting, receive UUV trail information, including position, velocity, attitude, angle velocity, acceleration, and transform the coordinate for these information; simulate the gyroscope and accelerometer measure principle to produce their output information, then, after sending the angle velocity and acceleration information out, enter the next simulation periods until the simulation finished.

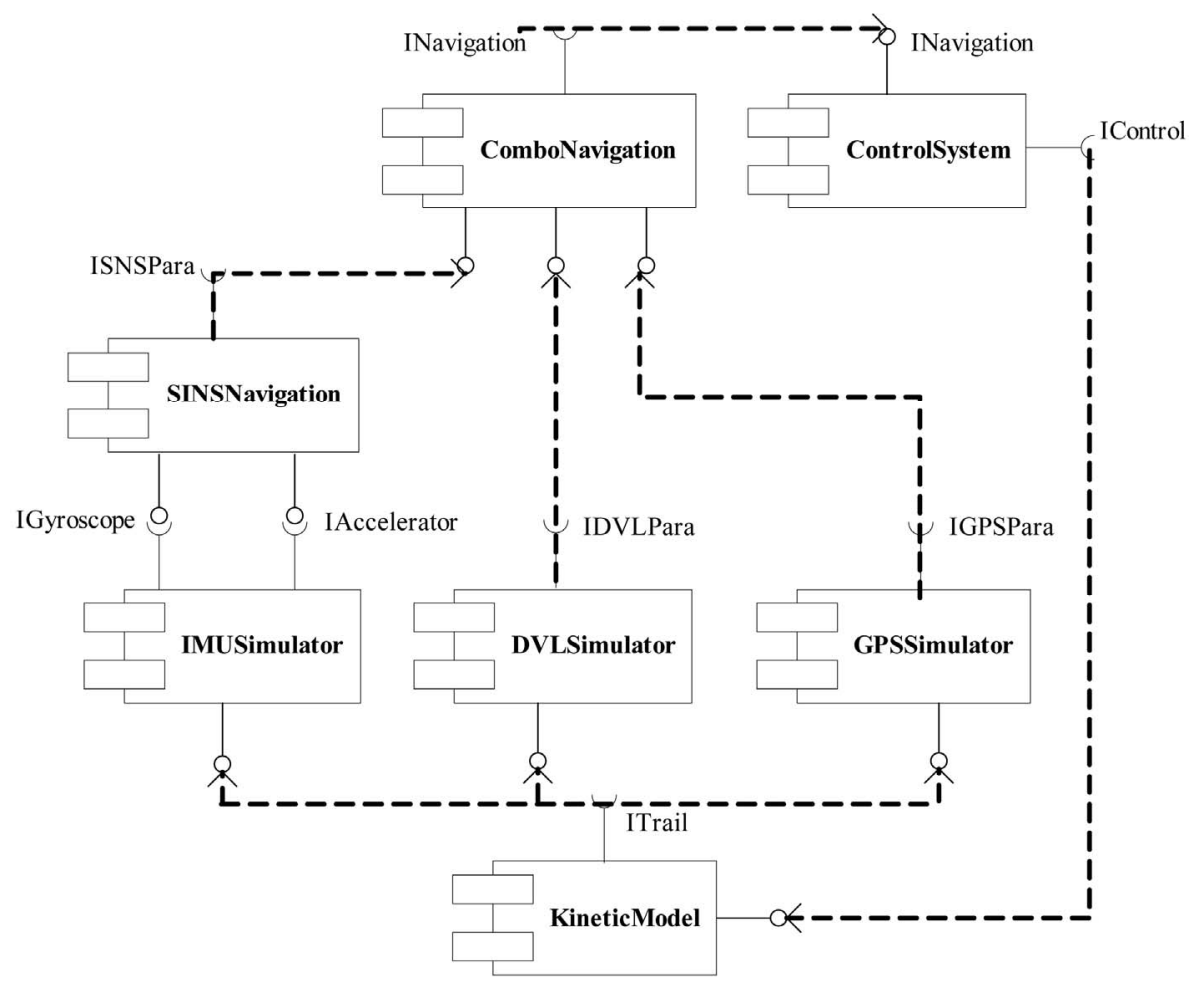

Figure 2. The simulation component diagram of the navigation and control integrative system.

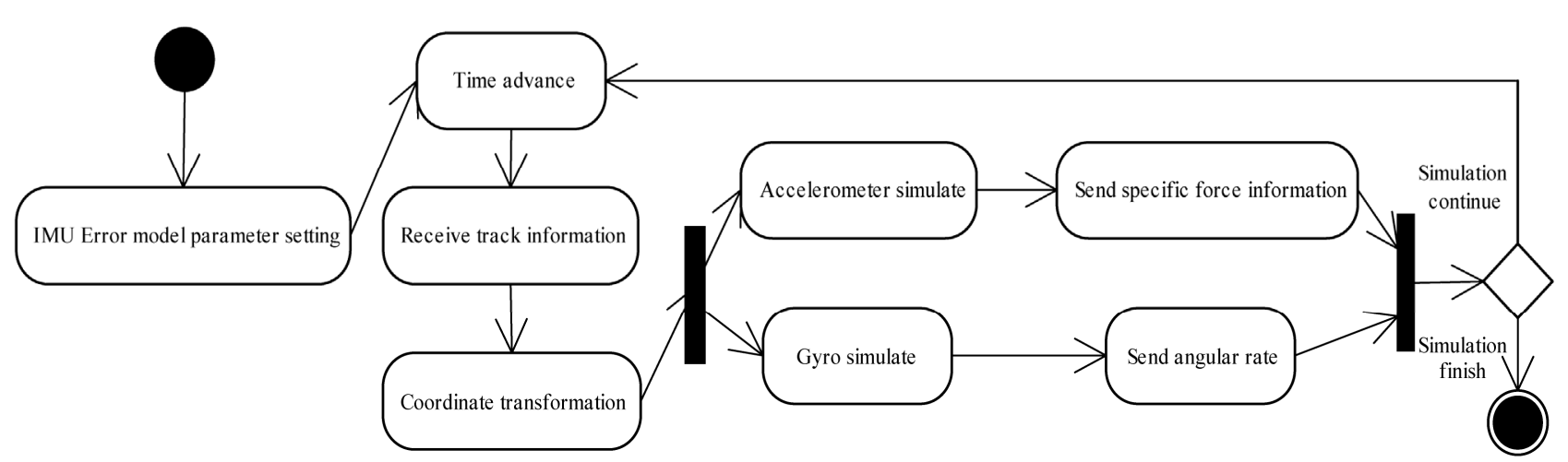

Figure 3. IMU component active diagram. 


\subsection{SINS Calculation Simulation Component}

SINS calculation simulation component can complete the calculation process of the strap-down inertial navigation based on the information supplied by gyroscope and accelerometer, it can be divided into four levels: first, component interface level, including SINS navigation information output interface (ISINSPara), event information interactive interface (IEvent), gyroscope information input interface (IGyroscope), accelerator information input interface (IAccelerator), initialization information input interface (IInitialization), integrated navigation information input interface (INavigation); second, component setting level, including the setting of initial alignment parameter (ParaInitAligment), initial parameter (ParaInit) and the time parameter (ParaTime); the third one is model calculation level that makes up of math function class (MathFunction), frame transform class (FrameClass), the SINS navigation calculation class (ISINSClass); and the last level is run management level that is composed by thread function (ThreadFunc), timer function (TimerFunc)and simulation operation class (SimRunClass).

The SINS simulation component active diagram is showed in Figure 4.

At first, SINS component should be initialized and the initial mission contains initial position, initial velocity and the initial alignment of the digital navigation platform; then calculates the attitude transformation matrix from body reference frame to navigation reference frame based on the angle velocity measured by gyroscope, extracts the attitude angle of UUV from the attitude transformation matrix, meanwhile, calculates the velocity of UUV by using both the attitude transformation matrix and the information from accelerometer, and the latitude and longitude of UUV is given by position matrix calculation; at last, exports the UUV navigation calculation information by the output interface.

\subsection{DVL Simulation Component}

DVL simulation component can simulate the working process of Doppler speed meter according to the UUV trail information, and produce the Doppler speed meter information, it contains four levels: first, component interface level, including trail information input interface (ITrail), event information interactive interface (IEvent), velocity information output interface (IVelocity), initialization information interface (IInitialization); second, component setting level, including the setting of initial parameter (ParaInit) and the time parameter (ParaTime); the third one is model calculation level that makes up of math function class (MathFunction), frame transform class (FrameClass), Doppler speed meter simulation class (DVLClass), Doppler error simulation class (DVLSensorErrClass); and the last level is run management level that is composed by thread function (ThreadFunc), timer function (TimerFunc) and simulation operation class (SimRunClass).

The DVL simulation component active diagram is showed in Figure 5.

After the start of the simulation component, the first thing is to finish the DVL error model parameter setting, then enter the simulation circle and request time promoting, receive UUV trail information and transform the coordinate; simulate the Doppler speed meter work principle to produce its output information and send the information to the integrated navigation simulation component, then, enter the next simulation periods until the simulation finished.

\subsection{GPS Simulation Component}

GPS simulation component can simulate the GPS satellites navigation system and provide the ephemeris data; itcan simulate the GPS receiver and choose the satellites, calculate the error, calculate the user's position and velocity according to the navigation message. GPS simulation component contains four levels: first, component interface level, including trail information input interface (ITrail), event information interactive interface (IEvent), GPS navigation information output interface (IGPSNavi-

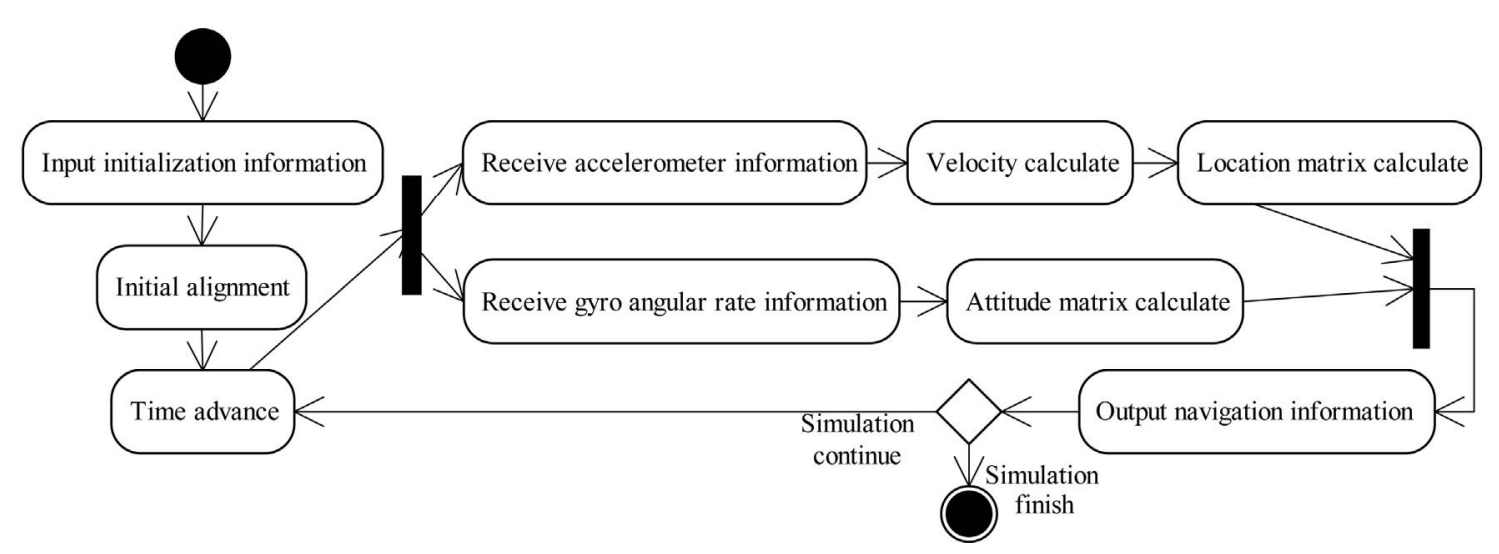

Figure 4. SINS calculation component active diagram. 
gation), initialization information input interface (IInitialization); second, component setting level, including the setting of initial parameter (ParaInit), system parameter (ParaSys) and the time parameter (ParaTime); the third one is model calculation level that makes up of math function class (MathFunction), frame transform class (FrameClass), noise produce class (NoiseClass), TLE data manage class (TLEDataClass), SDGP calculation class (SDGPClass), constellation simulation class (GPSSystemClass), GPS receiver simulation class (GPSReceiverClass); and the last level is run management level that is composed by thread function (ThreadFunc), timer function (TimerFunc) and simulation operation class (SimRunClass).

The GPS simulation component active diagram is showed in Figure 6.

At the beginning, GPS simulation component receives the initial information for initial setting, then enters the simulation circle, receives the trail information to simulate GPS constellation, produces the GPS ephemeris after
GPS error simulation, judges the visible star and adds the transmit error of the GPS signal to simulate the GPS receiver for pseudo-range and pseudo-range rate information, after uses GDOP for selecting stars, calculates GPS position and velocity information, then, sends the GPS measured data to the integrated navigation simulation component and enters the next simulation periods until the simulation finished.

\subsection{Integrated Navigation Simulation Component}

Integrated navigation simulation component is used to accomplish the fusion of all the navigation systems and give the integrated navigation results. It contains four levels: first, component interface level, including SINS navigation information input interface (ISINSPara), DVL navigation information input interface (IDVLPara), GPSnavigation information input interface (IGPSPara), integrated navigation information output interface (INavigation), event information interactive interface (IEvent),

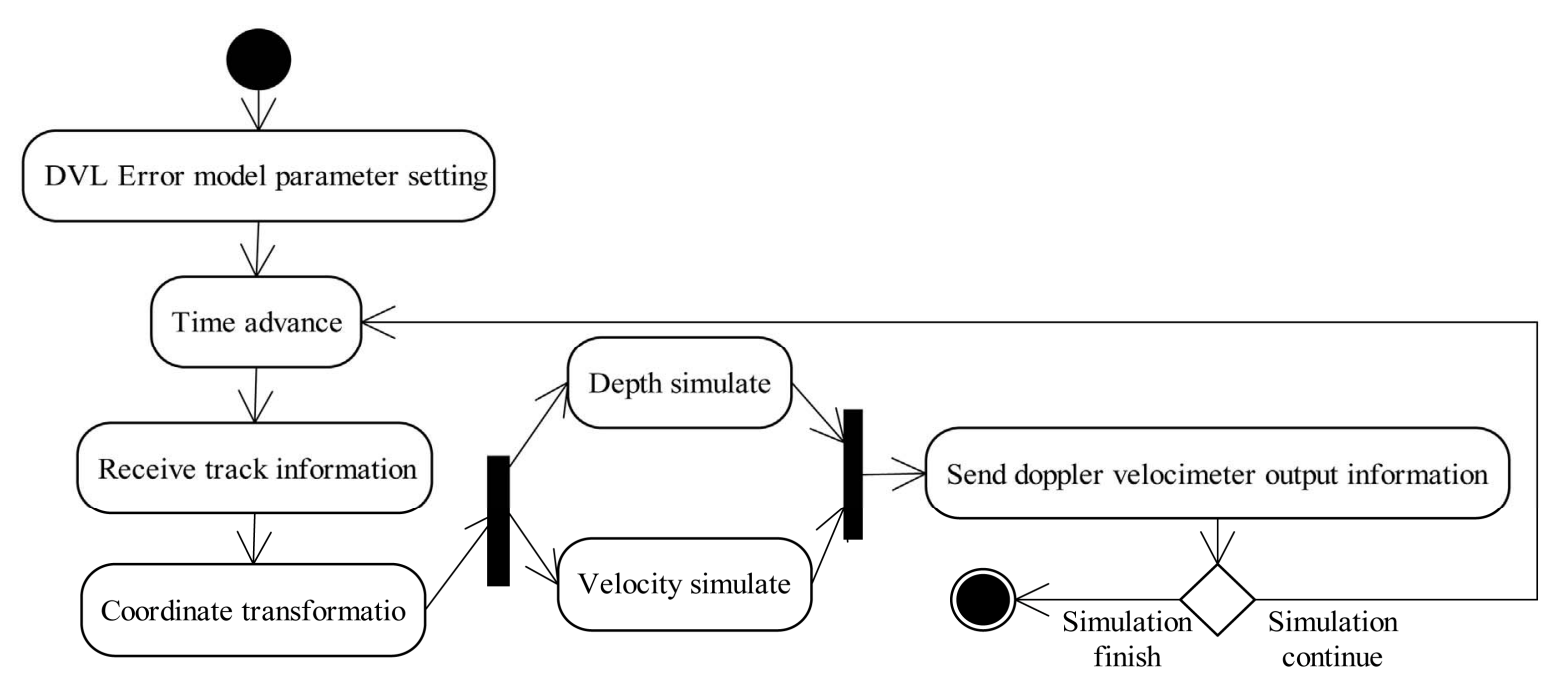

Figure 5. DVL simulation component active diagram.

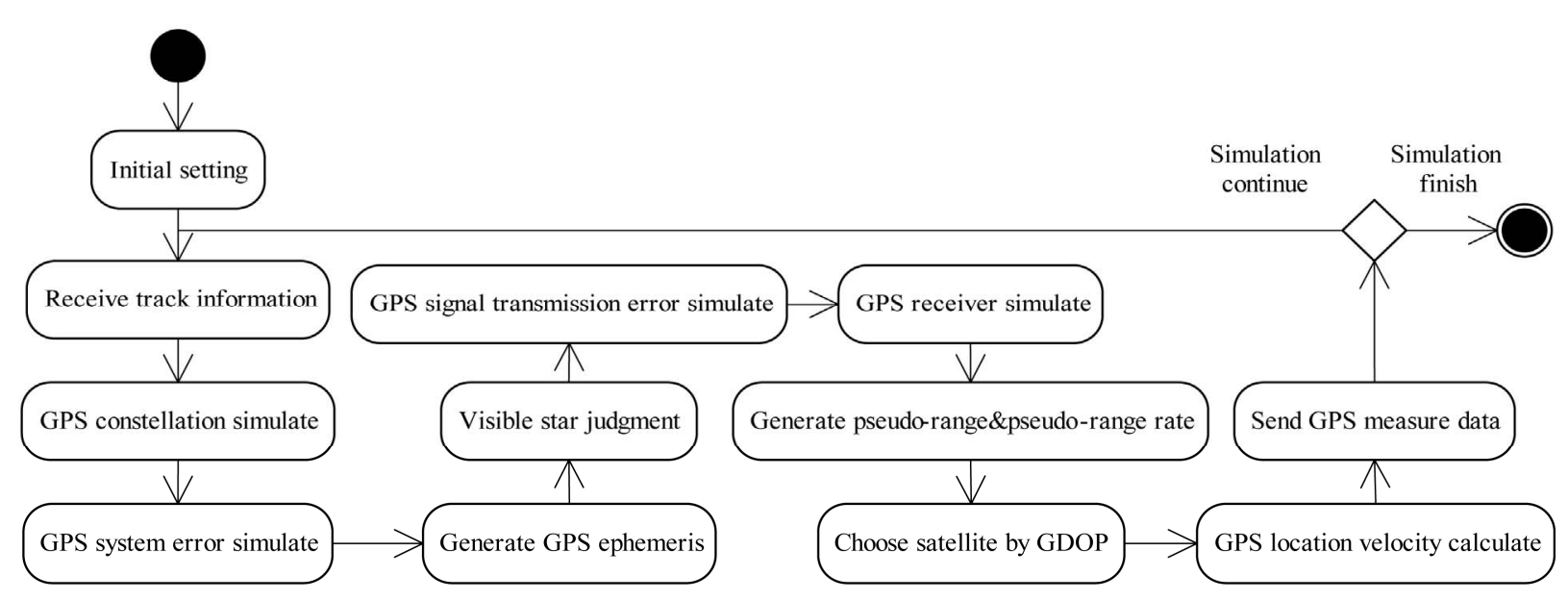

Figure 6. GPS simulation component active diagram. 
initialization information interface (IInitialization); second, component setting level, including the setting of integrated navigation parameter (ParaInit), system parameter (ParaSys) and the time parameter (ParaTime); the third one is model calculation level that makes up of math function class (MathFunction), frame transform class (FrameClass), SINS/GPS sub-filter class (SINSGPSClass), SINS/DVL sub-filter class (SINSDVLClass), information assignment class (InfoAssignmentClass), federal filter class (FederalFilterClass); and the last level is run management level that is composed by thread function (ThreadFunc), timer function (TimerFunc)and simulation operation class (SimRunClass).

The integrated navigation simulation component active diagram is showed in Figure 7.

Integrated navigation simulation component enters the integrated navigation calculation process after parameter and method setting, receives every navigation system's information through input interface according to the output frequency of their navigation parameters, then each sub-filter carry out calculation, then federal filter completes fusion calculation of the results from the subfilters by using given information assignment method, and sends the information to the control system component and SINS calculation component.

\section{Simulation Result}

Initializing the UUV attitude angles as $\theta=0^{\circ}, \psi=-90^{\circ}$, $\varphi=0^{\circ}$, and setting the initial position as latitude $20^{\circ} 0^{\prime} 0^{\prime \prime}$, longitude $120^{\circ} 0^{\prime} 0$ ", the initial velocity as $8 \mathrm{~m} / \mathrm{s}$, the initial depth as $20 \mathrm{~m}$ underwater; and the goal position as latitude $20^{\circ} 0^{\prime} 0$ ", longitude $120^{\circ} 30^{\prime} 0^{\prime \prime}$ and depth $60 \mathrm{~m}$ underwater. The modify periods is $12 \mathrm{~min}$ and it takes $3 \mathrm{~min}$ to float near the surface for receiving GPS signal. The whole voyage simulation curve is showed in the Figure 8.

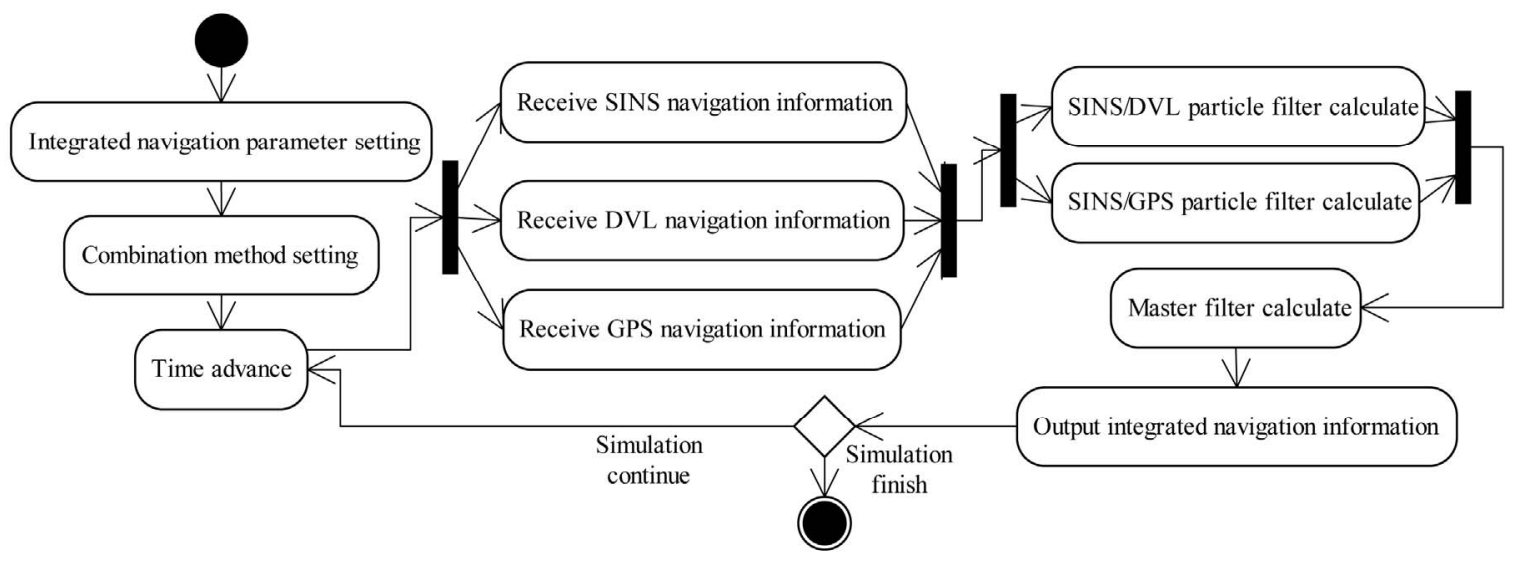

Figure 7. Integrated navigation simulation component active diagram.

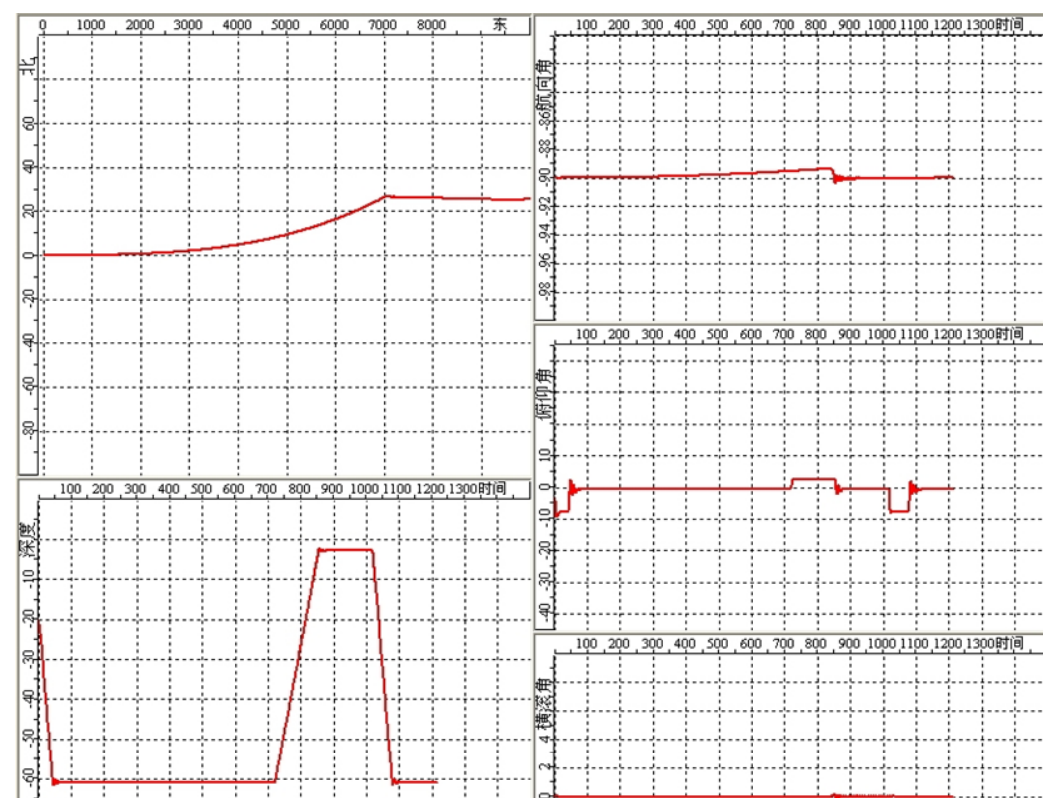

Figure 8. UUV move information view. 
This figure can be divided into five parts, the top left one is the motion trajectory in east and north plane, the migration of UUV is accumulated after launch, so, after 720 s voyage, it floats to the surface for GPS navigation modification, we can tell the modification of yaw angle from the top right curve. The bottom left curve shows how the depth changed. Firstly, UUV voyages in $-60 \mathrm{~m}$, and then floats upward to $-2 \mathrm{~m}$ to make GPS correction at $720 \mathrm{~s}$. After correction, UUV submerges to $-60 \mathrm{~m}$ and moves on. The curve in the right-middle shows the change of elevation angle, and the curve in the rightbottom shows the change of roll angles. From Figure 3 we can see the voyage simulation curve is similar with the scenario track.

\section{Conclusion}

This paper designs and realizes each simulation module of the UUV navigation and control integration simulation system which was analyzed, and describes the structure and working process of each module by activity diagrams. Through the validation of UUV integrated navigation whole process simulation, the simulation modules have well expansibility and reusability. And all of them can be reused in different simulation programs. It is beneficial to the development and maintenance of simulation program, and provides technical support of the development of the large comprehensive simulation system in the future.

\section{REFERENCES}

[1] C. J. J. Paredis, D. C. Antonio, R. Sinha, et al., "Composable Model for Simulation-Based Design,” Engineering with Computer, Vol. 17, No. 2, 2001, pp. 112-128. doi:10.1007/PL00007197

[2] C. J. Wan, J. R. Tan and A. Y. Liu, "Research on Component Prototype Modeling Based on Semantics," China Mechanical Engineering, Vol. 16, No. 16, 2005, pp. 1442 1446.

[3] P. Wang, B. H. Li, X. D. Chai, et al., "Simulation Component Based CGF System Architecture and Realization," Journal of System Simulation, Vol. 19, No. 5, 2007, pp. 1041-1044,

[4] Y. K. Wang, F. J. Kang, S. M. Duan and Y. H. Huang, "Design of Integrated Navigation Integrative Simulation System of AUV,” Journal of System Simulation, Vol. 21, No. 7, 2009, pp. 1874-1877. 